\title{
Facetas de desgaste dental en conductores de buses de una empresa de Medellín 2017
}

\section{Dental wear facets in drivers of buses of a company of Medellin 2017}

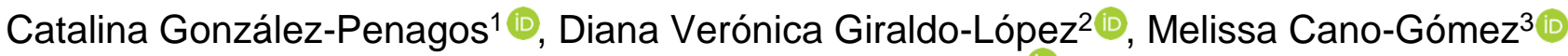 \\ Diana Isabel Ramírez-Pérez ${ }^{4}$ (D)
}

Tipología: Artículo de investigación científica y tecnológica

Para citar este artículo: González-Penagos C, Giraldo-López D, Cano-Gómez M, Ramírez-Pérez D. Facetas de desgaste dental en conductores de buses de una empresa de Medellín 2017. Duazary. 2019 septiembre; 16(3): 63 73. Doi: http://dx.doi.org/10.21676/2389783X.2969

Recibido en agosto 16 de 2018

Aceptado en marzo 22 de 2019

Publicado en línea en septiembre 01 de 2019

\section{RESUMEN}

El objetivo principal del estudio fue identificar las facetas de desgaste dental de los conductores de bus de una empresa de Medellín. Este fue un estudio de corte transversal, con un enfoque empírico analítico de alcance correlacional y de diseño no experimental. A través de un cálculo probabilístico se tomó una muestra de 278 conductores con un nivel de confianza del 98\%, un margen error del 5\% y una proporción esperada del 50\%. Cada participante diligenció una encuesta, y posteriormente se realizó una valoración clínica dental. A partir de los datos se hicieron análisis univariado, bivariado y multivariado. En los resultados hubo asociación estadísticamente significativa con la variable "ruta en la que conduce" $(\mathrm{p}=0,01)$, siendo la respuesta afirmativa a esta la única que se encuentra en la ecuación. Esta misma se presenta como factor protector (0,33 - IC 0,14-0,80). La mayoría de los conductores $(82,3 \%)$ manifestaron facetas de desgaste dental. En relación con la clasificación, el grado 1 fue el que más prevaleció. La razón del desgaste está dada por procesos multifactoriales, por lo que es muy importante detectar las facetas a tiempo para evitar un daño en la estructura dental.

Palabras clave: desgaste dental; salud bucal; transportes; trabajadores.

\footnotetext{
1. Fundación Universitaria Autónoma de las Américas. Medellín, Colombia. Correo: catalina.gonzalez@uam.edu.co http://orcid.org/0000-0002-7133-891X

2. Fundación Universitaria Autónoma de las Américas. Medellín, Colombia. Correo: diana.giraldo@uam.edu.co http://orcid.org/0000-0002-2976-9142

3. Fundación Universitaria Autónoma de las Américas. Medellín, Colombia. Correo: melissacanogomez@gmail.com http://orcid.org/0000-0001-7716-9689

4. Fundación Universitaria Autónoma de las Américas. Medellín, Colombia. Correo: diana.ramirez@ uam.edu.co http://orcid.org/0000-0003-1525-9878
} 


\section{ABSTRACT}

The main objective of this study was to identify the facets of dental wear of bus drivers in a company in Medellin. It was a cross-sectional study, with an empirical analytical approach of correlational scope and non-experimental design. Through a probabilistic calculation, a sample of 278 drivers was taken with a confidence level of $98 \%$, an error margin of 5\% and an expected proportion of 50\%. Each participant completed a survey and then a dental clinical assessment was made. Univariate, bivariate and multivariate analysis were made from the data. In the results there was a statistically significant association, with the variable route in which it leads $(p=0.01)$, with the affirmative response to this variable being the only one found in the equation. This is presented as a protective factor (0.33-CI 0.14-0.80). As a conclusion of this study, the great majority of drivers (82.3\%) presented facets of dental wear, and according to the classification, grade 1 was the most prevalent. The reason for the wear is given by multifactorial processes, so it is vital to detect them in time to avoid damage to the tooth structure.

Keywords: tooth wear; oral health; transportation; workers.

\section{INTRODUCCIÓN}

El desgaste de los dientes o pérdida de la estructura dental se relaciona estrechamente con la edad de las personas pues este es un proceso fisiológico que avanza a través del tiempo y se manifiesta por medio de facetas de desgaste. A pesar de que este es considerado un proceso normal asociado al envejecimiento dental y al uso a lo largo de la vida, se debe de tener especial control y cuidado cuando dicha pérdida estructural involucra la funcionalidad y la estética del paciente ${ }^{1}$, especialmente en aquellos casos que presentan mayor riesgo por sus actividades cotidianas, como es el caso de los conductores de buses.

Los conductores de transporte público pueden verse afectados por problemas digestivos $\mathrm{u}$ oculares, patologías en la cavidad bucal, sobrepeso y obesidad, retracciones musculares en los miembros inferiores y anormalidades de la columna lumbar, entre otros, además de la exposición a diversos elementos del medio ambiente tales como monóxido de carbono, plomo y ruido, que afectan de igual forma la salud $^{2}$. Con respecto a la salud bucal, el estilo de vida y las jornadas laborales que tiene este tipo de población hacen que el autocuidado sea complejo pues las condiciones de trabajo no permiten un tiempo adecuado para practicar hábitos como el del cepillado dental durante la jornada laboral; también el consumo de alimentos y frutos ácidos, el nivel de ansiedad y el estrés hacen que las condiciones para la salud bucal sean desfavorables, lo que produce una pérdida del tejido superficial del diente y da lugar a una faceta de desgaste que puede ser desde leve hasta severa, siendo esta última una situación que causa molestias e inconvenientes en el proceso de masticación, afectando así la alimentación y disminuyendo la calidad de vida de los conductores.

Las facetas de desgaste pueden tener diferentes grados o niveles como proceso normal de la masticación. Sin embargo, algunos factores como la dieta, el estilo de vida, la ocupación laboral, parafunciones, hábitos, cantidad de dientes, etc., pueden acentuar estos niveles de desgaste . $^{3}$.

El grado de atrición dental que puede presentarse depende de algunos factores como la fuerza de oclusión dada por el desarrollo de los músculos masticatorios. El fenómeno inicia como leves facetas muy suaves en las superficies dentales que hacen contacto durante la masticación; luego, a medida que pasa el tiempo, con la función dichas facetas aumentan de tamaño y se reduce la altura cuspídea, desapareciendo al mismo tiempo la anatomía incisal y oclusal de los dientes ${ }^{4}$.

En las civilizaciones antiguas el desgaste dental prevalecía; este era intenso y progresaba rápidamente. Dicha situación estaba relacionada con su alimentación. Los dientes, aparte de masticar, servían como herramientas de trabajo; además, los alimentos tenían diferentes consistencias, y muchos de estos se consumían crudos, lo que podía afectar la magnitud del desgaste dental. En la actualidad el modelo patológico triboquímico ha sustituido el modelo fisiológico abrasivo anterior. En las personas modernas, los factores dietéticos erosivos, el uso de implementos de higiene bucal abrasivos y contactos anormales de dientes debido a 
bruxismo son las principales razones para el desgaste dental ${ }^{5}$.

Cuando se habla de facetas de desgaste, se hace referencia al desgate mecánico que sufren las superficies incisales y oclusales de los dientes como resultado de fuerzas de rozamiento que modifican las superficies de los dientes implicados. En este proceso, tales superficies pasan de ser curvas a ser planas ${ }^{6}$, es decir, la pérdida de la estructura dental causada por el contacto de los dientes durante la oclusión y masticación puede afectar ambos tipos de denticiones, temporal y permanente. Las superficies más expuestas son las caras palatinas de los incisivos superiores y las caras vestibulares de los incisivos inferiores. Al realizar una inspección clínica, se observan superficies planas, brillantes y alargadas que coinciden con el patrón oclusal ${ }^{7}$.

Se han informado cambios adaptativos al sistema estomatognático en respuesta al desgaste, incluida la erupción continua, la ampliación del ciclo masticatorio, la remodelación de la articulación temporomandibular y el acortamiento de los arcos dentales debido a la migración dental ${ }^{8}$.

Teniendo en cuenta lo mencionado en apartados anteriores, el desgaste dental se define como la alteración de las superficies dentales, resultado del contacto mecánico de estas durante el proceso de la masticación o por parafunciones. Durante la trituración de los alimentos, los dientes contactan entre sí, ejerciendo fuerzas; sin embargo, esta función normal no es la única que puede producir desgaste de las superficies oclusales, pues apretar los dientes puede causar un desgaste a nivel local de estos en posición de máxima intercuspidación, ya sea en un acto de apretamiento constante $\mathrm{o}$ ante movimientos dentales pequeños ${ }^{9}$.

Teniendo en cuenta los factores de riesgo a los cuales se ve expuesta la población en estudio, el objetivo de esta investigación fue identificar las facetas de desgaste dental de los conductores de buses de una empresa de Medellín en 2017.

\section{MATERIALES Y MÉTODOS}

\section{Tipo de estudio}

Estudio cuantitativo descriptivo, de corte transversal con un alcance correlacional y de diseño no experimental.

\section{Población}

La población estuvo constituida por 560 conductores empleados por la empresa de transporte público de la ciudad de Medellín (Colombia).

\section{Muestreo}

Se efectuó un cálculo probabilístico de la muestra con un nivel de confianza del $98 \%$, un margen error del $5 \%$ y una proporción esperada del $50 \%$, dado que no existían estudios previos. Asimismo, la muestra fue estratificada por cada una de las rutas. Para seleccionar a los participantes que la conformarían, se realizó un muestreo aleatorio simple.

\section{Muestra}

El tamaño de la muestra probabilística y representativa de la población fue de $n=278$ conductores de buses de una empresa de transporte público. El estudio toma fuentes primarias de información cuya recolección fue entre los meses de junio y diciembre del año 2017 , en cada una de las terminales de buses.

\section{Criterios de inclusión y exclusión}

Se incluyeron en el estudio los trabajadores de una empresa de transporte público mayores de 18 años que ocupaban el cargo de conductores de buses, y fueron excluidos aquellos conductores con prótesis.

\section{Instrumento}

Se dispuso de una encuesta tipo formulario diligenciada por los conductores. En ella se abordaron temas sociodemográficos, laborales y de conocimientos en facetas de desgaste dental. 
Las variables tenidas en cuenta fueron: 1) Sociodemográficas: edad, sexo, nivel educativo, estrato socioeconómico, hijos, lugar de residencia y tipología familiar; 2) Factores laborales: tipo de conductor, ruta que conduce, tiempo de servicio en la empresa y tiempo de descanso; 3) Conocimiento en facetas de desgaste dental: "¿has percibido si aprietas o rechinas tus dientes?", "¿tienes dolor o fatiga en la sien o en los músculos de la cara?", "¿tienes dolor o fatiga en cuello u hombros?", "¿has experimentado dificultad para abrir ampliamente la boca?”, "¿consideras que tus dientes se han desgastado muy rápido últimamente?”, “ ¿sientes que descansas, independientemente de las horas que hayas dormido?".

Se realizó una valoración clínica dental a cada participante. Para esto se estandarizaron dos investigadoras odontólogas utilizando la medida de concordancia del índice Kappa ${ }^{10}$, que indicó un grado de acuerdo de 0,84.

Para la valoración clínica, se tuvo en cuenta el índice de facetas de desgaste dental según Hansson y Nilner, modificado por el Dr. Carlos Matta $^{6}$. Este parámetro se clasificó así: Grado 0 : ausencia de facetas de desgaste dentario; Grado 1: faceta de desgaste dentario en esmalte; Grado 2: facetas de desgaste dentario en esmalte e islotes de dentina hasta $1 \mathrm{~mm}$; Grado 3: 1/3 incisal desgastado, con facetas de desgaste dentario exageradas hacia lingual o bucal; Grado 4: facetas de desgaste dentario que comprometen la cámara pulpar. En particular, cuando el grado de pérdida de tejido dental se concentra en los grados 3 y 4 , se desarrollan patologías que influyen en la supervivencia de los dientes y causan problemas estéticos, funcionales $\mathrm{o}$ de sensibilidad $^{11}$.

Los exámenes se llevaron a cabo en las instalaciones del lugar de trabajo donde se encontraba el conductor, en un espacio abierto, ventilado, iluminado, con luz artificial y la ayuda de una linterna minera para la cabeza como fuente de iluminación directa.

\section{Análisis estadístico}

Los datos se analizaron a partir de la revisión de las encuestas. El análisis univariado incluyó las variables sociodemográficas, laborales, de conocimientos de desgaste y facetas de desgaste. Las técnicas de información que se utilizaron en este caso fueron las medidas de frecuencia, porcentajes, mediana, moda, rango intercuartil y valor mínimo y máximo.

Para el análisis bivariado se tuvo en cuenta la prueba estadística chi cuadrado $\left(\mathrm{X}^{2}\right)$ y un nivel $\mathrm{p}$ de significancia menor a $0,05^{12}$. Por otra parte, para el análisis multivariado se incluyeron las variables en las que hubo confusión y que tuvieron significancia en el análisis bivariado. Para estas últimas se hizo una regresión logística con fines explicativos (método Enter). Finalmente, se digitalizó y procesó la información en el paquete estadístico PRISM ${ }^{\circledR}$ (versión 6.07, junio 12 de 2015), con licencia de la Fundación Universitaria Autónoma de las Américas.

\section{Declaración sobre los aspectos éticos}

La presente investigación tuvo en cuenta la Resolución 8430 de 1993 del Ministerio de la Salud y Protección Social del Estado colombiano $^{13}$ y se clasifica con riesgo mínimo. En línea con esta norma (en particular, el capítulo 1, artículo 14, donde se resalta la importancia de un acuerdo por escrito), a cada participante se le dio a conocer un consentimiento informado donde podía autorizar su participación en la investigación. Por último, este proyecto contó con el aval del Comité de Ética en Investigación institucional (CEI) (Acta 29 del 31 de enero de 2017).

\section{RESULTADOS}

Se contó con la participación de 278 conductores de buses cuya edad promedio fue 36,53 años ( $\mathrm{DE}=11,86$ años). La edad mínima de los encuestados fue de 18 años, y la máxima edad reportada fue 68 , lo que representa una mediana en la edad de 35 años, en tanto que la edad más frecuentemente reportada fue 23 años. De los participantes, el 99,6\% (277) eran 
hombres, y el 0,36\% (1) era mujer. En cuanto al estado civil, el 38,1\% (106) convivían en unión libre; el 29,1\% (81) eran casados y el $28,4 \%$ (79) eran solteros. El nivel de escolaridad predominante fue bachillerato completo, con el $45,3 \%(126)$ y el $29,1 \%$ (81) no terminaron el bachillerato, mientras que el $18,7 \%$ (52) tenían primaria completa e incompleta; solo el 6,84\% (19) tenían un nivel educativo que oscilaba entre técnico, tecnólogo y universitario incompleto.

En la Tabla 1 se presentan las distribuciones porcentuales de las diferentes respuestas de los factores laborales de los conductores de cuatro rutas de buses de la ciudad de Medellín. Es de resaltar que en su mayoría los conductores son fijos y descansan una vez a la semana.

Tabla 1. Distribución porcentual de las respuestas de los factores laborales de los conductores.

\begin{tabular}{|c|c|c|}
\hline Variable & Frecuencia absoluta & $\begin{array}{c}\text { Porcentaje } \\
(\%)\end{array}$ \\
\hline \multicolumn{3}{|c|}{ Tipo de conductor } \\
\hline Conductor fijo & 225 & 80,9 \\
\hline Conductor auxiliar & 52 & 18,7 \\
\hline \multicolumn{3}{|l|}{ Ruta que conduce } \\
\hline Ruta 1 & 56 & 20,2 \\
\hline Ruta 2 & 46 & 16,6 \\
\hline Ruta 3 & 73 & 26,3 \\
\hline Ruta 4 & 102 & 36,8 \\
\hline \multicolumn{3}{|c|}{ Tiempo de servicio en la empresa } \\
\hline Promedio años de servicio & 9,5 años & \\
\hline \multicolumn{3}{|c|}{ Tiempo de descanso } \\
\hline 1 vez a la semana & 116 & 41,7 \\
\hline 2 veces a la semana & 49 & 17,6 \\
\hline Cada 15 días & 84 & 30,2 \\
\hline Otro & 29 & 10,4 \\
\hline
\end{tabular}

En relación con los conocimientos que tenían los conductores frente al desgaste dental, se identificó que el 51,4\% (143) ha percibido que aprieta o rechina sus dientes; el $75 \%$ (207) expresa no presentar dolor o fatiga en la sien o músculos de la cara; el 60\% (167) manifiesta sentir dolor o fatiga en el cuello y los hombros; $y$ el $84,7 \%$ (233) dice no experimentar dificultad para abrir ampliamente la boca. Del total de conductores, el 47,1\% (130) considera que sus dientes se han desgastado muy rápido últimamente.

Respecto al cansancio, se halló que el 44,9\% (125) de los conductores siente que no descansa, independientemente de las horas que haya dormido; así, el 72,3\% (201) afirmó sentirse cansado y con sueño durante el día.
En cuanto al desgaste y la edad de los 278 conductores examinados, se verificó que el $82,3 \%$ (229) contaba con facetas de desgaste y que la faceta de desgaste grado 1 estaba presente en todos los grupos de edad, distribuida de la siguiente manera: el 29\% (80) son conductores desde los 18 hasta los 30 años; el 25,5\% (71), entre 31 y 43 años; el 19\% (53), de 44 a 56 años; y el 4,6\% (13), mayores de 57 años.

En términos de prevalencia de facetas de desgaste según grado de desgaste, se observó que, de las 5.350 piezas con facetas de desgaste, 1.949 se presentaron en los incisivos. El $64,9 \%$ (1.263) de estos tenían grado 1; $28,3 \%$ (553) eran grado $2 ; 6,7 \%$ (131) fueron grado 3 ; y el $0,1 \%$ (2) correspondió a grado 4 (Tabla 2). 
Tabla 2. Prevalencia de grado de desgaste según grupo de dientes

\begin{tabular}{|c|c|c|c|c|}
\hline \multirow{2}{*}{$\begin{array}{c}\text { Grado de } \\
\text { desgaste }\end{array}$} & \multicolumn{4}{|c|}{ Grupos de dientes } \\
\cline { 2 - 5 } & Incisivos (\%) & Caninos (\%) & Premolares (\%) & Molares (\%) \\
\hline Grado 1 & 64,9 & 66 & 88,5 & 93,3 \\
\hline Grado 2 & 28,3 & 28,7 & 9,4 & 5,7 \\
\hline Grado 3 & 6,7 & 5,2 & 2,1 & 0,6 \\
\hline Grado 4 & 0,1 & 0,1 & & 0,4 \\
\hline
\end{tabular}

Con respecto a los caninos, se constató desgaste en 1.016 piezas, de las cuales el $66 \%$ (671) fueron grado $1 ; 28,7 \%$ (291), grado 2; $5,2 \%$ (53), grado $3 ;$ y $0,1 \%$ (1), grado 4 (Tabla 2).

En los premolares, el desgaste se manifestó en 1.257 piezas: el $88,5 \%$ (1.112) fueron grado 1 ; el $9,4 \%$ (118), grado 2 ; y el $2,1 \%$ (27), grado 3 . En cuanto a los molares, el desgaste estaba presente en 1.128 piezas, distribuidas así: 93,3\% (1.053) grado $1,5,7 \%$ (64) grado 2, $0,6 \%$ (7) grado 3 y $0,4 \%$ (4) grado 4 (Tabla 2).
Una vez analizado cada sextante, se encontró que en todos los sextantes predominó el desgaste grado 1. Específicamente, para el sextante 1 (dientes $17,16,15,14)$ se presentó en el 76,62\% (213); para el sextante 2 (de canino 13 al 12,11, 21, 22, 23), en el 61,15\% (170); para el sextante 3 (dientes 24, 25, 26, 27 ), en el $60,43 \%$ (168); para el sextante 4 (dientes 37, 36, 35, 34), en el 61,51\% (171); para el sextante 5 (dientes 33,32, 31, 41, 42, 43), en el 64,03\% (178); y finalmente, para el sextante 6 (dientes 44, 45, 46, 47), en el $51,44 \%$ (143) (Tabla 3). ). 
Tabla 3. Distribución de las facetas por sextantes de los conductores de buses de una empresa de la ciudad de Medellín

\begin{tabular}{|c|c|c|c|}
\hline Variable & Definición & $\begin{array}{l}\text { Frecuencia } \\
\text { absoluta }\end{array}$ & $\begin{array}{l}\text { Porcentaje } \\
(\%)\end{array}$ \\
\hline \multicolumn{4}{|l|}{$\begin{array}{l}\text { Sextante } 1 \\
(\text { dientes } 17,16,15,14)\end{array}$} \\
\hline Desgaste grado 1 & Compromiso de esmalte & 213 & 76,62 \\
\hline Desgaste grado 2 & Compromiso de esmalte y dentina & 9 & 3,24 \\
\hline No presentó desgaste & & 56 & 20,14 \\
\hline \multicolumn{4}{|c|}{$\begin{array}{l}\text { Sextante } 2 \text { (dientes } 13,12,11, \\
21,22,23)\end{array}$} \\
\hline Desgaste grado 1 & Compromiso de esmalte & 170 & 61,15 \\
\hline Desgaste grado 2 & $\begin{array}{l}\text { Compromiso de esmalte y dentina } \\
\text { hasta } 1 \mathrm{~mm}\end{array}$ & 78 & 28,06 \\
\hline Desgaste grado 3 & Tercio incisal está desgastado & 12 & 4,32 \\
\hline No presentó desgaste & & 18 & 6,47 \\
\hline \multicolumn{4}{|l|}{$\begin{array}{l}\text { Sextante } 3 \\
\text { (dientes } 4,25,26,27 \text { ) }\end{array}$} \\
\hline Desgaste grado 1 & Compromiso de esmalte & 168 & 60,43 \\
\hline Desgaste grado 2 & $\begin{array}{l}\text { Compromiso de esmalte y dentina } \\
\text { hasta } 1 \mathrm{~mm}\end{array}$ & 8 & 2,88 \\
\hline No presentó desgaste & & 102 & 36,69 \\
\hline \multicolumn{4}{|c|}{$\begin{array}{l}\text { Sextante } 4 \text { (dientes } 37,36,35 \text {, } \\
34 \text { ) }\end{array}$} \\
\hline Desgaste grado 1 & Compromiso de esmalte & 171 & 61,51 \\
\hline Desgaste grado 2 & $\begin{array}{l}\text { Compromiso de esmalte y dentina } \\
\text { hasta } 1 \mathrm{~mm}\end{array}$ & 16 & 5,76 \\
\hline No presentó desgaste & & 91 & 32,73 \\
\hline \multicolumn{4}{|c|}{$\begin{array}{l}\text { Sextante } 5 \\
\text { (dientes } 33,32,31,41,42,43 \text { ) }\end{array}$} \\
\hline Desgaste grado 1 & Compromiso de esmalte & 178 & 64,03 \\
\hline Desgaste grado 2 & $\begin{array}{l}\text { Compromiso de esmalte y dentina } \\
\text { hasta } 1 \mathrm{~mm}\end{array}$ & 77 & 27,7 \\
\hline Desgaste grado 3 & Tercio incisal está desgastado & 12 & 4,32 \\
\hline No presentó desgaste & & 1 & 3,96 \\
\hline \multicolumn{4}{|l|}{$\begin{array}{l}\text { Sextante } 6 \\
\text { (dientes } 44,45,46,47)\end{array}$} \\
\hline Desgaste grado 1 & Compromiso de esmalte & 143 & 51,4 \\
\hline Desgaste grado 2 & $\begin{array}{l}\text { Compromiso de esmalte y dentina } \\
\text { hasta } 1 \mathrm{~mm}\end{array}$ & 8 & 2,88 \\
\hline No presentó desgaste & & 127 & 45,68 \\
\hline
\end{tabular}

Al analizar la posible asociación de algunas características sociodemográficas con las facetas de desgaste, se halló que existe una correlación positiva $(0,234$ - valor $\mathrm{p}=0,000)$ entre la edad y el promedio general de desgaste. Esto indica que, a mayor edad, mayor desgaste. Así se encontró asociación entre la variable "facetas de desgaste" con el grupo de edad $\left(X^{2} 26,462 p=0,000\right)$. En este mismo sentido se observó una relación con la variable que indagaba si el conductor consideraba que los dientes se le habían desgastado muy rápido últimamente $\left(\mathrm{X}^{2} 11,005 \mathrm{p}=0,004\right)$.

Teniendo en cuenta los anteriores resultados, además de vincular el criterio de HosmerLemeshow, se llevó a cabo un análisis multivariado con el fin de identificar cuáles variables formarían parte de la ecuación. De esta manera se agregaron al modelo de regresión logística variables como estado civil $(\mathrm{p}=0,072)$, nivel de escolaridad $(\mathrm{p}=0,090)$, ruta en la que conduce $(\mathrm{p}=0,043)$, percepción que 
tiene de apretar o rechinar los dientes $(\mathrm{p}=0,025)$ y si alguien le había comentado al conductor si apretaba o rechinaba los dientes $(\mathrm{p}=0,050)($ Tabla 4$)$.

Tabla 4. Factores sociodemográficos, laborales y de conocimientos de desgaste dental

\begin{tabular}{|l|c|c|}
\hline \multicolumn{1}{|c|}{ Variable } & Prueba X2 & Valor p \\
\hline Estado civil & 17,1 & 0,07 \\
\hline Nivel de escolaridad & 16,36 & 0,09 \\
\hline Ruta en la que conduce & 15,96 & 0,04 \\
\hline Percepción que tiene de apretar o rechinar los dientes & 7,36 & 0,02 \\
\hline Alguien le ha comentado si apretaba o rechinaba los dientes & 6,00 & 0,05 \\
\hline
\end{tabular}

Los resultados arrojados por la regresión logística muestran una asociación estadísticamente significativa con la variable "ruta en la que conduce" $(\mathrm{p}=0,014)$, siendo la respuesta afirmativa a esta variable la única que se encuentra en la ecuación. Esta misma se presenta como factor protector $(0,339$ - IC $0,142-0,807)$.

\section{DISCUSIÓN}

La pérdida de esmalte dental es un proceso fisiológico que ocurre a través del tiempo. Sin embargo, debe considerarse patológico cuando el grado de la pérdida crea alteraciones a nivel funcional y estético, acompañadas por síntomas. La abrasión, la atrición, la erosión y la abfracción son cuatro de las causas que ocasionan la pérdida de estructura dental ${ }^{7}$.

El desgaste afecta tanto a las superficies oclusales como a las incisales de los dientes. La atrición fisiológica por la masticación prevalece en cada persona; sin embargo, su severidad depende de factores como la dieta, pues si esta contiene sustancias abrasivas puede causar mayor pérdida. Dicha situación puede incluso empeorar si se tiene bruxismo o defectos de estructura en el esmalte o la dentina ${ }^{4}$. También depende de la fuerza de oclusión teniendo en cuenta el desarrollo de los músculos masticatorios.

El desgaste inicia como pequeñas superficies lisas y pulidas en las zonas del diente que hacen contacto durante la trituración de alimentos, formándose unas pequeñas facetas. Luego, con la función estas facetas se vuelven mucho más grandes y afectan el tamaño de la cúspide, perdiéndose al mismo tiempo la anatomía dental ${ }^{4}$.

La atrición es la pérdida de tejido dental que se da por el contacto diente-diente durante la función. Impacta en ambos tipos de dentición y afecta en su mayoría a las superficies palatinas de los incisivos superiores y a las caras vestibulares de los incisivos inferiores. Al realizar el examen clínico se observan facetas de desgaste planas, lisas, brillantes y alargadas que coinciden con el patrón oclusal. No es común que se dé una exposición pulpar o excesiva sensibilidad a nivel de la dentina, ya que la pérdida de estructura dental es muy lenta, lo que permite la aposición de dentina secundaria-reparativa ${ }^{7}$.

El término "bruxismo" es derivado del francés la bruxomanie, utilizado por primera vez por Marie Pietkiewicz (1907), pero se le acredita a Frohman (1931) la utilización de esta palabra para identificar un problema dentario desencadenado por el movimiento mandibular anormal. Se usan diferentes términos relativos al acto de bruxar y apretar los dientes; tal es el caso de bruxismo, bruxismo en céntrica, bruxismo en excéntrica, bruxismo nocturno, bruxismo diurno, bruxomanía, parafunción, apretamiento dentario, rechinamiento dentario, parasomnia, etc. Carlsson y Magnusson $^{14}$ 
definen "parafunción" como una actividad que no tiene propósitos funcionales $\mathrm{y}$ "apretamiento-rechinamiento" como el acto de apretar y frotar los dientes entre sí, conociéndose ambos como bruxismo ${ }^{15}$.

Los resultados del presente estudio indican una prevalencia de $82,3 \%$ (229) de facetas de desgaste, lo que guarda relación con lo reportado por la clínica especializada de la Universidad San Martín de Porres, quienes hallaron facetas de desgaste en el 77,5\% de pacientes evaluados ${ }^{16}$. El resultado es similar al de la Facultad de Odontología de la Universidad de Cuenca, donde examinaron a 151 estudiantes y la prevalencia de desgaste dental fue del $77,5 \%(177)^{17}$.

En este estudio se encontró que, de acuerdo a la edad, el grupo más afectado por las facetas de desgaste fue el comprendido entre los 18-30 años, el cual representó el 39\% (108), seguido por el grupo entre 31-43 años, equivalente al $31 \%$ (87). Estos hallazgos son similares a los del estudio de la Universidad San Martín de Porres ${ }^{16}$, donde el grupo más afectado fue el que se hallaba entre los 18-33 (34,74\%), seguido por el de 34-49 años $(31,05 \%)$. Ahora bien, los resultados son diferentes a los señalados por el Policlínico Universitario Raúl Sánchez, en el municipio Pinar del Río, donde el grupo más afectado fue el de 31-40 años, que representa un $30 \%$ del total ${ }^{4}$.

En el presente estudio se descubrió que el $51,4 \%$ (143) ha percibido que aprieta o rechina sus dientes; resultado diferente al observado en Estados Unidos entre 84 sujetos previamente diagnosticados con disfunciones temporomandibulares, donde el $32,1 \%$ reportó alguna actividad de bruxismo ${ }^{18}$.

Respecto a la pieza más afectada por las facetas de desgaste, en el presente estudio el resultado fue para los incisivos con el 36,4\% (1949), similar resultado reportado en Lima, Perú, con el $(48,26 \%)$ también en los incisivos ${ }^{16}$.

En el estudio del Policlínico Universitario Raúl Sánchez, en el municipio Pinar del Río ${ }^{4}$, se evidenció que el grado de desgaste que más prevaleció en la muestra estudiada fue el grado 2 , representado por un $76,7 \%$ del total. En esta investigación se encontró, además, que la faceta de desgaste grado 1 estaba presente en todos los grupos de edad.

Finalmente, se concluye que la gran mayoría de conductores $(82,3 \%)$ presentaron facetas de desgaste dental $\mathrm{y}$, de acuerdo con la clasificación, el grado 1 fue el de mayor prevalencia. Se considera que la razón del desgaste está asociada a procesos multifactoriales, por lo que es de suma importancia la detección temprana para evitar un daño mayor en la estructura dental. Por otro lado, cabe resaltar que durante la valoración clínica se identificaron algunos problemas de salud bucal tales como enfermedad periodontal, caries dental, edentulismo parcial, halitosis, acúmulos considerables de placa bacteriana y cálculo dental, todos estos asociados a prácticas deficientes e inadecuadas del cepillado y uso de seda dental. En consecuencia, a partir de estos resultados se implementará una estrategia para contribuir al mejoramiento de la salud bucal de los participantes.

\section{DECLARACIÓN SOBRE CONFLICTOS DE INTERESES}

Los autores no presentan conflicto de interés en la publicación de este artículo.

\section{CONTRIBUCIÓN DE LOS AUTORES}

CG, directora del proyecto. CG, DG y MC participaron en la concepción del estudio. CG, DG, MC, y DR participaron en la ejecución del proyecto, análisis e interpretación de los datos y en la redacción del artículo, y aportaron a la revisión crítica del contenido intelectual. Todas aprobaron la versión final.

\section{REFERENCIAS BIBLIOGRÁFICAS}

1. Koksal T, Dikbas I, Kazaoglu E. Alternative Restorative Approach for Treatment of Patient with Extremely Worn Dentition. The New York State Dental Journal [revista en la internet]. 2009 [citado 2018 Oct 24]; 75(5):525.

Disponible 
https://www.researchgate.net/profile/Temel_ Koksal/publication/38061017_Alternative_res torative_approach_for_treatment_of_patient_ with_extremely_worn_dentition/links/552e04 a50cf29b22c9c51142/Alternative-restorativeapproach-for-treatment-of-patient-withextremely-worn-dentition.pdf

2. Chaparro P, Guerrero J. Condiciones de Trabajo y Salud en Conductores de una Empresa de Transporte Público Urbano. Rev. Salud Pública [revista en la internet]. 2001 [citado 2018 Oct 24]; 3(2):171-187. Disponible en: http://www.scielo.org.co/scielo.php?script=sc i_arttext\&pid=S0124$00642001000200005 \& \operatorname{lng}=\mathrm{en}$

3. Benmehdi S, Rioboo M, Bourgeois D, Sanz M. Lesiones cervicales no cariosas y su asociación con la periodontitis. Rev. Periodoncia y Osteointegración [revista en la internet]. 2009 [citado 2018 Oct 24]; 19(3). Disponible en: http://www.sepa.es/images/stories/SEPA/RE VISTA_PO/articulos.pdf/19-3_04.pdf

4. García J, Carmona J, González X, González $\mathrm{R}$, Labrador M. Atrición dentaria en la oclusión permanente. Rev. Ciencias Médicas [revista en la internet]. 2014 [citado 2018 Oct 24]; 18(4):566-573. Disponible en: http://scielo.sld.cu/scielo.php?script=sci_artte $\mathrm{xt} \& \mathrm{pid}=\mathrm{S} 1561-31942014000400003 \& \operatorname{lng}=\mathrm{es}$

5. D'Incau E, Couture C, Maureille B. Human tooth wear in the past and the present: tribological mechanisms, scoring systems, dental and skeletal compensations. Archives of oral biology [revista en la internet]. 2012 [citado 2018 Oct 24]; 57(3):214-29. Disponible en: https://www.sciencedirect.com/science/article /pii/S0003996911002755

6. Rendón A. Facetas de desgaste dentario y personalidad: revisión bibliográfica. Kiru [revista en la internet]. 2015 [citado 2019 Feb 28]; 12(1):88-90. Disponible en: http://www.aulavirtualusmp.pe/ojs/index.php/ Rev-Kiru0/article/viewFile/466/381

7. Armas A, Latorre C, Pallenzona M, Guiza E. Desgaste dental y factores de riesgo asociados. Rev CES Odontología [revista en la internet]. 2010 [citado 2018 Oct 24]; 23(1). Disponible en: http://revistas.ces.edu.co/index.php/odontolog ia/article/view/755

8. Kaidonis J. Tooth wear: the view of the anthropologist. Clinical oral investigations [revista en la internet]. 2007 [citado 2018 Oct 24]; 12(1). Disponible en: https://www.ncbi.nlm.nih.gov/pmc/articles/P MC2563149/

9. Ferro K, Morgano S, The Glossary of. prosthodontic Terms. Foundation, The Academy of Prosthodontics. The Journal Of Prosthetic Dentistry [revista en la internet]. 2005 [citado 2018 Oct 24]; 94(1). Disponible en:

http://www.academyofprosthodontics.org/_Li brary/ap_articles_download/GPT9.pdf

10. López G, Fernández P. Medidas de concordancia: el índice de Kappa. Unidad de Epidemiología Clínica y Bioestadística. Complexo Hospitalario-Universitario Juan Canalejo. A Coruña (España) Cad Aten Primaria 1999 [citado 2018 Oct 24]; (6):169171. Disponible en: https://www.fisterra.com/mbe/investiga/kappa /kappa2.pdf

11. Lee A, He L, Lyons K, Swain M. Tooth wear and wear investigations in dentistry. Journal of Oral Rehabilitation [revista en la internet]. 2012 [citado 2018 Oct 24]; 39(1). Disponible en: https://www.ncbi.nlm.nih.gov/pubmed/21923 888

12. Londoño F. Metodología de la investigación epidemiológica. Quinta edición. Bogotá: Manual Moderno, 2014 [citado 2018 Oct 24]; 112-27.

13. Colombia. Ministerio de Salud. Resolución 8430 de 1993 por la cual se establecen las normas científicas, técnicas y administrativas para la investigación en salud. [En línea]. [Citado 2017 Dic 5]. Disponible en: https://www.minsalud.gov.co/sites/rid/Lists/B ibliotecaDigital/RIDE/DE/DIJ/RESOLUCIO N-8430-DE-1993.PDF. 
14. Carlsson G, Magnusson T. Bruxism and other oral parafunctions. Management of Temporomandibular Disorders in the General Dental Practice. Quintessence Publ Co Inc. 1999 [citado 2018 Oct 24]; (5):33-42.

15. Díaz O, Estrada B, Franco G, Espinoza C, González R, Badillo E. Lesiones no cariosas: atrición, erosión, abrasión, abfracción, bruxismo. Revista Oral [revista en la internet]. 2011 [citado 2018 Oct 24]; 12(38). Disponible en:

http://www.medigraphic.com/pdfs/oral/ora2011/ora1138d.pdf

16. Vergiú C, Malatto J, Berger C, Torres L, Saberbein J, Bautista G, et al. Prevalencia de facetas de desgaste. Kiru [revista en la internet]. 2012 [citado 2018 Oct 24]; 9(1). Disponible http://www.usmp.edu.pe/odonto/servicio/201 2/Kiruv.9/Kiru_v.9_Art9.pdf.

17. Castillo J, Ramón A. Prevalencia de desgaste dental en estudiantes de la Facultad de Odontología de la Universidad de Cuenca. [Tesis de pregrado]. 2017. Disponible en: http://dspace.ucuenca.edu.ec/bitstream/12345 6789/26289/1/TESIS.pdf

18. Pergamalian A. Rudy T. The association between wear facets, bruxism, and severity of facial pain in patients with temporomandibular disorders. The Journal of Prosthetic Dentistry, [revista en la Internet]. 2003 [citado 2018 Oct 24]; 90(2): 194-200. Disponible en: https://www.ncbi.nlm.nih.gov/pubmed/12886 214 